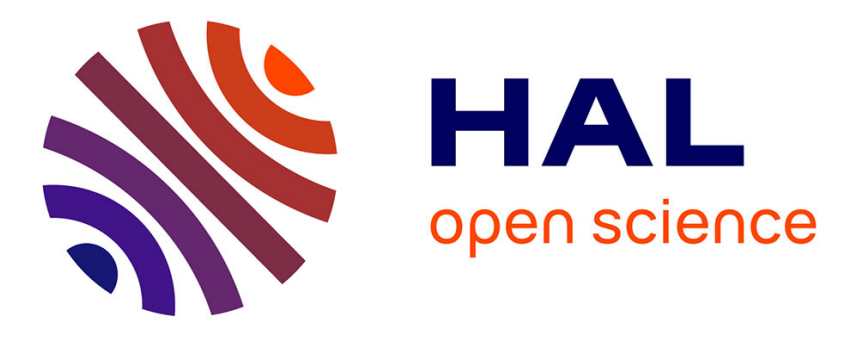

\title{
Time-resolved measurement of concentration fluctuations in a confined bubbly flow by LIF
}

Elise Alméras, Sébastien Cazin, Véronique Roig, Frédéric Risso, Frédéric Augier, Cécile Plais

\section{- To cite this version:}

Elise Alméras, Sébastien Cazin, Véronique Roig, Frédéric Risso, Frédéric Augier, et al.. Time-resolved measurement of concentration fluctuations in a confined bubbly flow by LIF. International Journal of Multiphase Flow, 2016, vol. 83, pp. 153-161. 10.1016/j.ijmultiphaseflow.2016.03.011 • hal-01332255

\section{HAL Id: hal-01332255 \\ https://hal.science/hal-01332255}

Submitted on 15 Jun 2016

HAL is a multi-disciplinary open access archive for the deposit and dissemination of scientific research documents, whether they are published or not. The documents may come from teaching and research institutions in France or abroad, or from public or private research centers.
L'archive ouverte pluridisciplinaire HAL, est destinée au dépôt et à la diffusion de documents scientifiques de niveau recherche, publiés ou non, émanant des établissements d'enseignement et de recherche français ou étrangers, des laboratoires publics ou privés. 


\section{Open Archive TOULOUSE Archive Ouverte (OATAO)}

OATAO is an open access repository that collects the work of Toulouse researchers and makes it freely available over the web where possible.

This is an author-deposited version published in : http://oatao.univ-toulouse.fr/ Eprints ID : 15871

To link to this article : DOI:10.1016/j.ijmultiphaseflow.2016.03.011 URL : http://dx.doi.org/10.1016/j.ijmultiphaseflow.2016.03.011

To cite this version : Alméras, Elise and Cazin, Sébastien and Roig, Véronique and Risso, Frédéric and Augier, Frédéric and Plais, Cécile Time-resolved measurement of concentration fluctuations in a confined bubbly flow by LIF. (2016) International Journal of Multiphase Flow, vol. 83. pp. 153-161. ISSN 0301-9322

Any correspondence concerning this service should be sent to the repository administrator: staff-oatao@,listes-diff.inp-toulouse.fr 


\title{
Time-resolved measurement of concentration fluctuations in a confined bubbly flow by LIF
}

\author{
Elise Alméras ${ }^{\mathrm{a}, \mathrm{b}, *}$, Sébastien Cazin ${ }^{\mathrm{a}}$, Véronique Roiga ${ }^{\mathrm{a}}$, Frédéric Risso ${ }^{\mathrm{a}}$, Frédéric Augier ${ }^{\mathrm{b}}$, \\ Cécile Plais ${ }^{\mathrm{b}}$ \\ a Institut de Mécanique des Fluides de Toulouse (IMFT), Université de Toulouse, CNRS-INPT-UPS, Toulouse, France \\ ${ }^{\mathrm{b}}$ IFP Energies nouvelles, Rond-point de l'échangeur de Solaize, BP 3, Solaize 69360, France
}

Keywords:

Confined bubbly flow

Mixing

LIF and spectrometry

\begin{abstract}
A B S T R A C T
The present work investigates the mixing of a low-diffusivity dye in a swarm of bubbles at high Reynolds number confined in a Hele-Shaw cell for gas volume fractions ranging from 1.4 to $5.4 \%$. A patch of a fluorescent dye is injected within the swarm and, during its mixing, its concentration is measured at a given location in an observation volume of $4.5 \mathrm{~mm}^{2}$ by means of Laser Induced Fluorescence at a frequency of $250 \mathrm{~Hz}$. A spectrometer is used to analyse the light issued from the observation volume and to distinguish the fluoresced light from other light sources. Simultaneously, the bubble distribution around the observation volume is imaged with a high speed camera synchronised with the spectrometer in order to assess the LIF technique in bubbly flow. Thanks to the good time resolution, rapid and intense concentration fluctuations corresponding to dye patches passing through the observation volume are recorded and are superimposed to a slow global evolution. This slow global evolution shows first an increase of the concentration and then an exponential decrease due to the mixing by bubble-induced agitation. This exponential decay, which is incompatible with a diffusion process, is consistent with the transport by dye capture in bubbles wakes that are quickly dampened by the shear-stress at the walls. The one-point statistics of the concentration fluctuations (probability density function and spectrum) also point out that mixing in a confined bubbly flow is intermittent and convective.
\end{abstract}

\section{Introduction}

Bubbles columns are commonly used for chemical and biological processes to enhance the mixing of chemical species by the bubble rising motions without using mechanical devices. Macroscopic mixing times are usually estimated by correlating tracer concentrations measured on the global system or on several locations (Pandit and Joshi, 1983). Macroscopic description of mixing has limitations. For instance, recently, McClure et al. (2015), have pointed out the difficulty to identify the impact of the measurement location on the macroscopic mixing time. A local description of mixing in bubbly flows is thus preferable but is still missing. Even the main mechanisms remain unclear. This lack of knowledge mainly comes from the difficulties to perform precise concentration measurements in bubbly flows. A solution to get around this problem consists in studying mixing in an array of fixed spheres mimicking a bubble swarm (Besnaci, 2012; Besnaci et al., 2010).

\footnotetext{
* Corresponding author at: Physics of Fluids Group, Faculty of Science and Technology, University of Twente, P.O. Box 217, 7500 AE Enschede, The Netherland.

E-mail address: e.o.almeras@utwente.nl (E. Alméras).
}

White and Nepf (2003) and Tanino and Nepf (2008) have also studied mixing in random arrays of obstacles in the context of environmental fluid mechanics. These studies underline the presence of two main mechanisms involved in the mixing of a weakly diffusive dye in flows at relative high Reynolds numbers. The first one is due to the direct interactions of the dye with the obstacles. In particular the dye can be caught in the wakes and also strongly twisted. The second mixing mechanism is the specific turbulence induced by interactions between the wakes (Amoura, 2008; Risso et al., 2010; Riboux et al., 2013). In a bubbly flow, the two mixing mechanisms described previously are still present and the mobility of the bubbles has to be taken into account too. The relative contributions of these various mechanisms may depend on the flow configuration, on the structure of the wakes and of the random agitation in the liquid phase. In a bubbly flow confined in a thin gap, where turbulence cannot develop, mixing turns out to result from dye capture in the wakes and to be incompatible with a diffusion process (Bouche et al., 2013). On the contrary mixing in a three-dimensional homogeneous bubbly flow is due to turbulence induced by bubbles and can be modelled by an equivalent Fick law (Alméras et al., 2015). Up to now the description of mixing 
in a bubbly flow was restricted to low acquisition frequency and no measurement of concentration fluctuations has been performed as far as we know. The issue is however important to have deeper knowledge of mixing mechanisms.

The purpose of this study is twofold. From the point of view of the metrology, we introduce an original experimental technique devoted to local concentration measurements in a bubbly flow at high acquisition frequency. This technique is based on Laser Induced Fluorescence (LIF) and uses a spectrometer in order to distinguish the fluoresced light from other light sources. Previous techniques used in our team to investigate confined bubbly flows are based on a global lighting of the cell, which prevents them from being applied to three-dimensional bubble columns because of the optical light occultation caused by the bubbles. Since the present technique only considers a small measurement volume by means of the use of optical fibres, its extension to three dimensional cases is possible. Some light distortions of the concentration field are nevertheless still present. In the present work, we have focused on a confined bubbly flow in order to be able to simultaneously use the new local measurement technique together with a flow visualization by means of a camera, which is only possible in a two-dimensional column. By this way, it has been possible to develop a signal processing method that allowed us to remove unambiguously the spurious parts of the concentration signal which were perturbed by bubbles passing close or throughout the measurement volume. Thanks to this method, reliable measurements of concentration fluctuations within a confined bubble swarm have been obtained, and for the first time, PDFs and time spectra of concentration fluctuations have been measured in a bubbly flow. The application of the present technique to a three-dimensional geometry is left for a future work.

From the point of view of the physics, the results have revealed the role of the intermittent transport by the bubbles and explained why the mixing in a two-dimensional bubbly flow cannot be described by a pure diffusion process.

The paper is organised as follows. The Section 2 presents the experimental set-up while the Section 3 describes the instrumentation used to measure the concentration. In Section 4 the validation procedure of the concentration measurement is described. A physical analysis of mixing in a confined bubbly flow is performed in Section 5 and conclusions are given in Section 6.

\section{Experimental set-up}

The mixing experiments take place in a thin cell which is presented in Fig. 1. The cell is composed of two glass plates of $400 \mathrm{~mm}$ wide, $800 \mathrm{~mm}$ high and separated by a gap of width $w=1 \mathrm{~mm}$. It is filled with an aqueous solution of magnesium sulfate $\left(\mathrm{MgSO}_{4}\right)$. Salt of magnesium is first dissolved in distilled water at a concentration of $5 \times 10^{-2} \mathrm{~mol} / \mathrm{L}$ and mixed up using a magnetic stirrer. The solution is then filtered in order to remove the residual solid. Adding a small amount of salt avoids the bubbles coalescence without affecting significantly the physical properties of the distilled water (Bouche et al., 2013). The liquid is initially at rest and bubbles are generated by capillary tubes of inner diameter $0.6 \mathrm{~mm}$ which are regularly distributed at the bottom of the cell. The gas injectors are connected to a large enough pressurized tank in order to provide a stationary gas flow rate, which is varied to adjust the gas volume fraction from 1 to $5.4 \%$. The averaged twodimensional equivalent diameter of the bubbles $d=\sqrt{4 A} / \pi$ (where $A$ is the projected area of the bubble on the cell plan) varies from $3.9 \pm 0.4 \mathrm{~mm}$ to $4.2 \pm 0.4 \mathrm{~mm}$ as the gas volume fraction increases in this range (Bouche et al., 2012). The bubble diameter is therefore large compared to the gap size $\left(\frac{w}{d} \ll 1\right)$ and bubbles are flattened. By varying the gas volume fraction, the Reynolds number defined by $R e=\frac{\rho V d}{v}$ (where $V$ is the rising velocity of a single

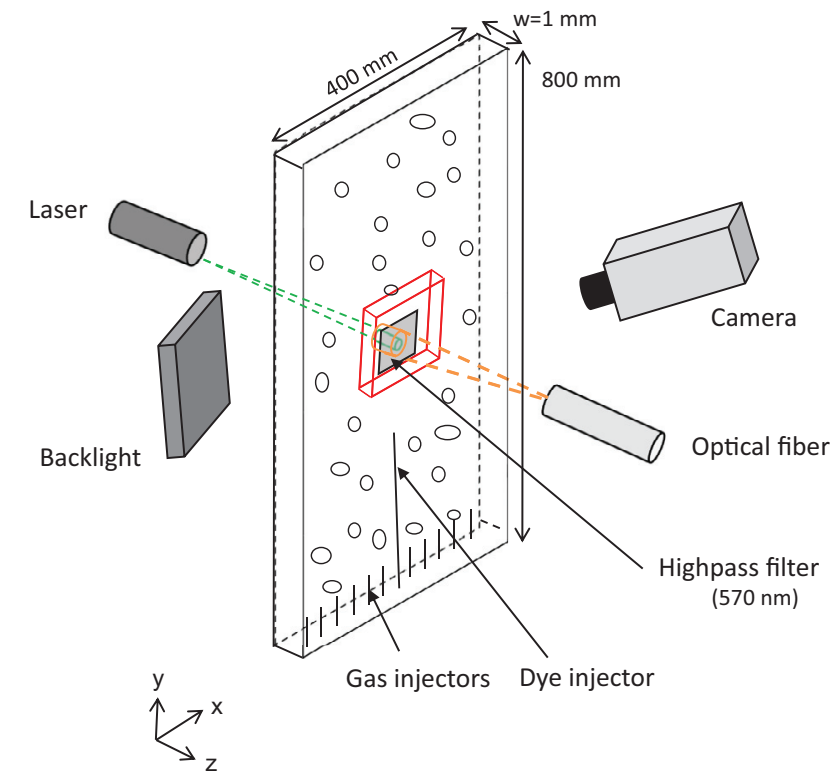

Fig. 1. Experimental set-up and instrumentation. The lighted volume is denoted by the small cylinder (in green), the observation volume by large cylinder (in orange), the field of view of the camera by the parallelepiped (in red). (For interpretation of the references to colour in this figure legend, the reader is referred to the web version of this article.)

bubble, $\rho$ is the density of the liquid and $v$ its kinematic viscosity) evolves from 450 to 500 and the Reynolds number based on the gap $\operatorname{Re}\left(\frac{w}{d}\right)^{2}$ goes from 25 to 30 . Since $\operatorname{Re} \gg 1$ and $\operatorname{Re}\left(\frac{w}{d}\right)^{2} \gg 1$, the flow is dominated by inertia and thus differs from the classic Hele-Shaw regime. The liquid perturbations induced in the bubble wakes are localised just behind the bubbles and are strongly attenuated by the shear stress at sidewalls (Roig et al., 2012). In that case, collective instability and turbulence cannot develop due to the confinement (Bouche et al., 2014).

Mixing experiments are carried out $400 \mathrm{~mm}$ above the gas injectors where the gas volume fraction is homogeneously distributed and the bubbles have reached their terminal velocity. They consist in injecting a passive fluorescent dye within the swarm through a long capillary tube of inner diameter $0.6 \mathrm{~mm}$ (Fig. 1). A volume of $1 \mathrm{~mL}$ of rhodamine WT at an initial concentration $c_{0}=10^{-4} \mathrm{~mol} / \mathrm{L}$ is injected during $2 \mathrm{~s}$ by a volumetric pump. During the injection, a jet of dye is generated and dampens approximately in around one viscous time, $T_{v}=\frac{w^{2}}{v}=1 \mathrm{~s}$. After attenuation of the jet, mixing induced by bubbles can be studied (Fig. 2). The time evolution of the concentration in an observation volume of $4.5 \mathrm{~mm}^{3}$ is measured using Laser Induced Fluorescence (LIF) and spectrometry. For 3 gas volume fractions ( $\alpha=1.4,2.8$ and 5.4\%), concentration signals are recorded at 3 different distances $H$ from the dye injector tip $(H=5,10$ and $15 \mathrm{~cm})$. For each case, 5 experiments have been performed to improve the statistical convergence of the results.

It is worth mentioning that the advective transport of dye in the cell is mainly achieved by a velocity field parallel to the plane of the cell depending on the coordinate in the direction perpendicular to that plane. In particular, the friction at the walls and the velocity gradient throughout the gap play a major role in the structure of the bubble wakes as shown by Roig et al. (2012), Bouche et al. (2014) and Filella et al. (2015). Within a viscous time scale $w^{2} /(4 v)$, the in-gap velocity profile generated by a bubble passage evolves from a plug flow profile to a Poiseuille flow profile with a maximum velocity decreasing exponentially with time. The present study is devoted to study the mixing in such a flow and should not be confused with a bidimensional flow extending to infinity. 


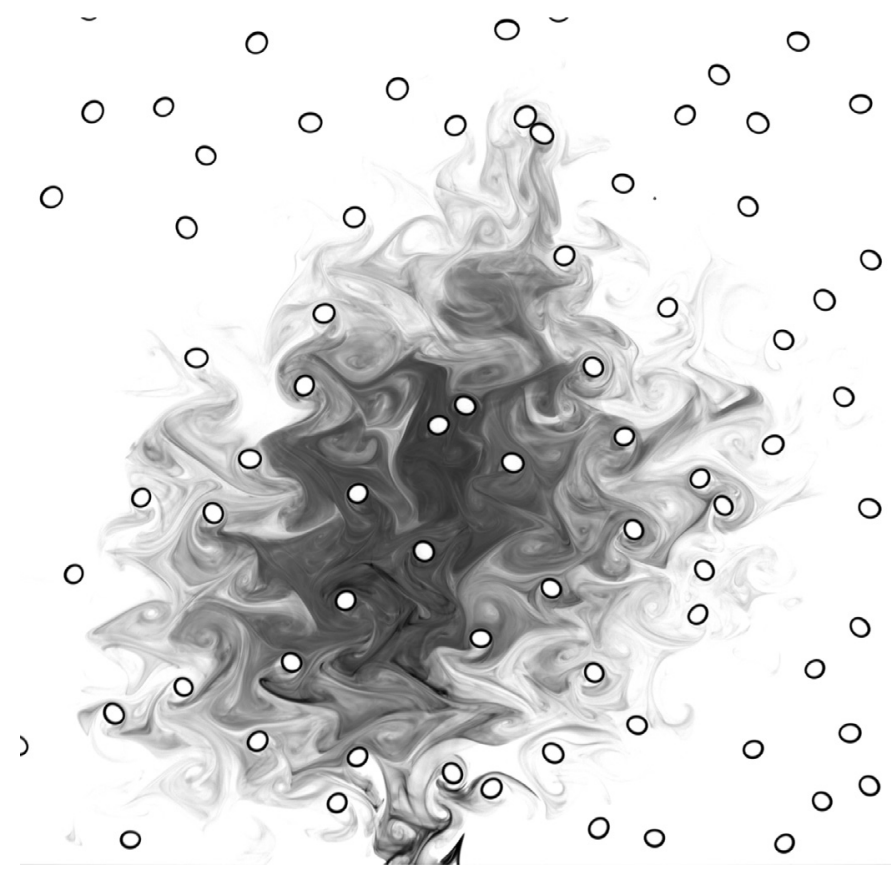

Fig. 2. Image of the mixing process. $\alpha=5.3 \%$.

Moreover, the measurement technique described below doesn't give any information about the distribution of the dye in the depth direction. It only provides a measurement of the concentration averaged over the gap.

\section{Concentration measurement by LIF and spectrometry coupled with imaging}

Measurement of concentration in bubbly flows by optical techniques must be carefully checked to avoid optical bias due to the presence of bubbles. Concentration measurements have, thus, been carried out by means of two independent and synchronized systems that provide complementary information about the precise meaning of the measured signals.

The first one is based on LIF measured by spectrometry. A fluorescent dye, the rhodamine WT, is excited by a continuous laser of wavelength $532 \mathrm{~nm}$ (YAG532, $10 \mathrm{M}$, Lasiris) placed on one side of the cell (Fig. 1). The laser beam illuminates a cylindrical volume of $0.5 \mathrm{~mm}$ diameter and $1 \mathrm{~mm}$ depth through the cell which is called hereafter the lighted volume. The absorption spectrum of the rhodamine WT ranges from 480 to $590 \mathrm{~nm}$ and its emission spectrum ranges from 560 to $680 \mathrm{~nm}$ (Fig. 3). The fluoresced light is then collected in a cylindrical volume of $2.4 \mathrm{~mm}$ diameter and $1 \mathrm{~mm}$ depth named observation volume by an optical fibre disposed on the other side of the cell (Fig. 1). As the laser beam is directly pointed in the direction of the optical fibre, a high-pass filter with a cut-off wavelength of $570 \mathrm{~nm}$ (OG570, Melles Griot) is placed between the cell wall and the optical fibre to protect the spectrometer sensor from the laser beam. The optical fibre is connected to a spectometer USB2000+ developed by Ocean Optics. The spectrometer is used to measure the spectrum of the collected light $E(\lambda, t)$ at each time $t$ in the range of wavelengths $\lambda$ from 340 to $1030 \mathrm{~nm}$ with a resolution of $0.3 \mathrm{~nm} /$ pixel and a maximal acquisition frequency of $1 \mathrm{kHz}$. Practically, our measurements were recorded at an acquisition frequency equal to $250 \mathrm{~Hz}$ with an integration time equal to $4 \mathrm{~ms}$ in order to get enough photons to ensure a reliable measurement. The concentration of the dye is then calculated by integrating, for each time step, the spectrum of the collected light in a range of wavelengths included in the emission spectrum of rhodamine WT (Fig. 3). By using a spectrometer, the range of

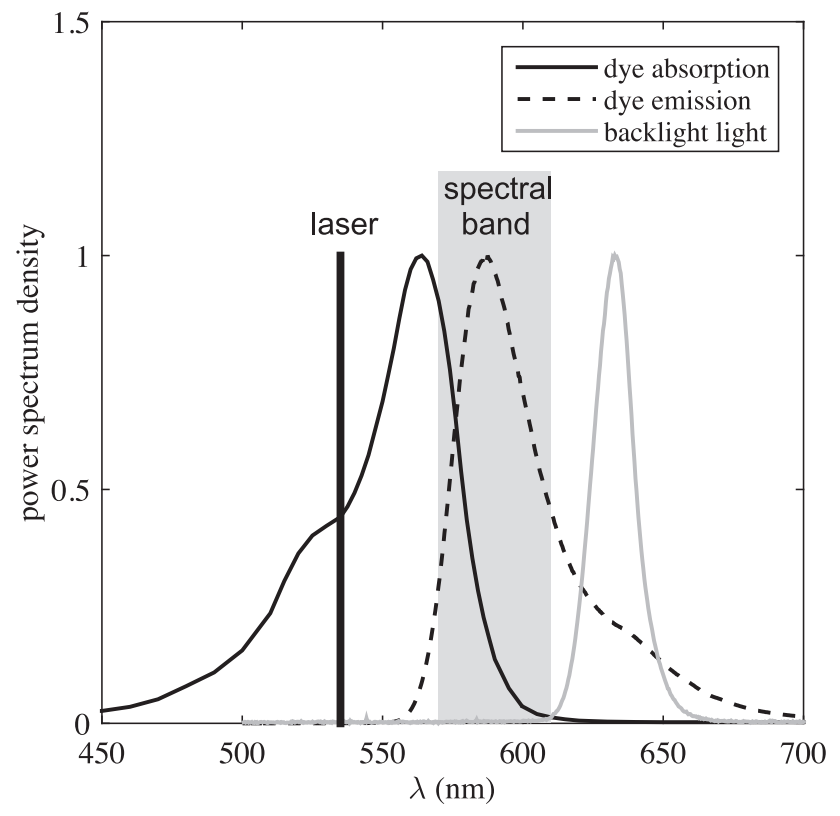

Fig. 3. Absorption and emission spectra of rhodamine WT, and emission spectrum of the led panel used for the imaging technique. The marked spectral band corresponds to the part of the spectrum of the collected light used to determine the concentration measured by spectrometry.

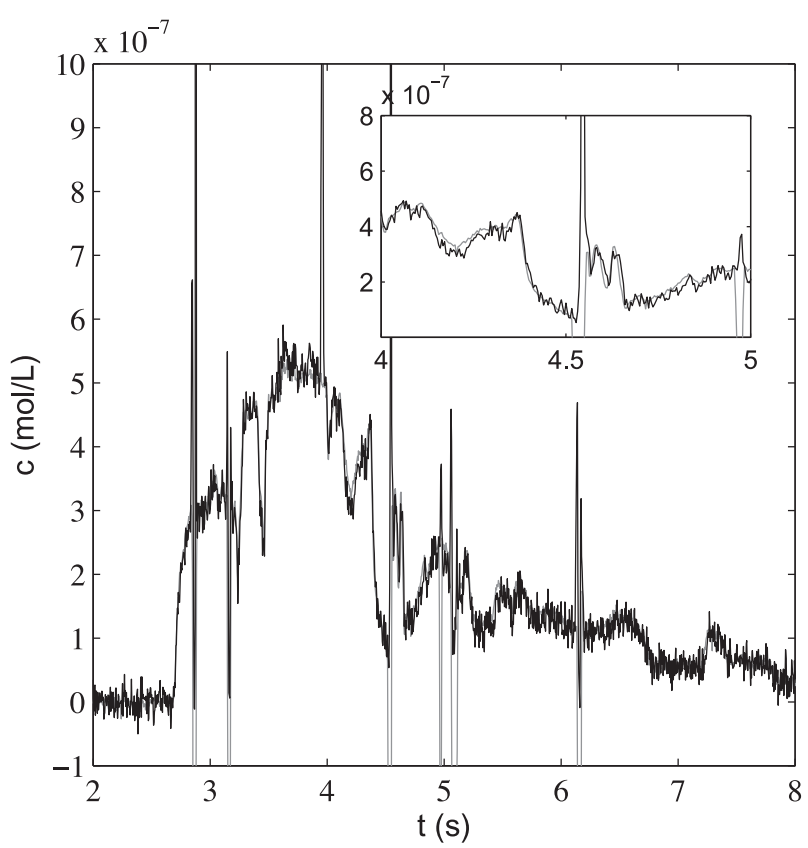

Fig. 4. Comparison of the signals measured by the spectrometer (black) and by imaging (gray). $\alpha=3.7 \% . H=5 \mathrm{~cm}$.

wavelengths for the integration of the emission spectrum can be selected very precisely and can even be chosen afterwards. In practice, the considered range is restricted to $570-610 \mathrm{~nm}$ because of the presence of the red LED pannel used to visualize the flow field around the observation volume (Fig. 3). Provided that the dye concentration is sufficiently low (see next section for more details), the instantaneous concentration is calculated by the following expression:

$c(t)=K \int_{\lambda=570 \mathrm{~nm}}^{\lambda=610 \mathrm{~nm}} E(\lambda, t) d \lambda$ 

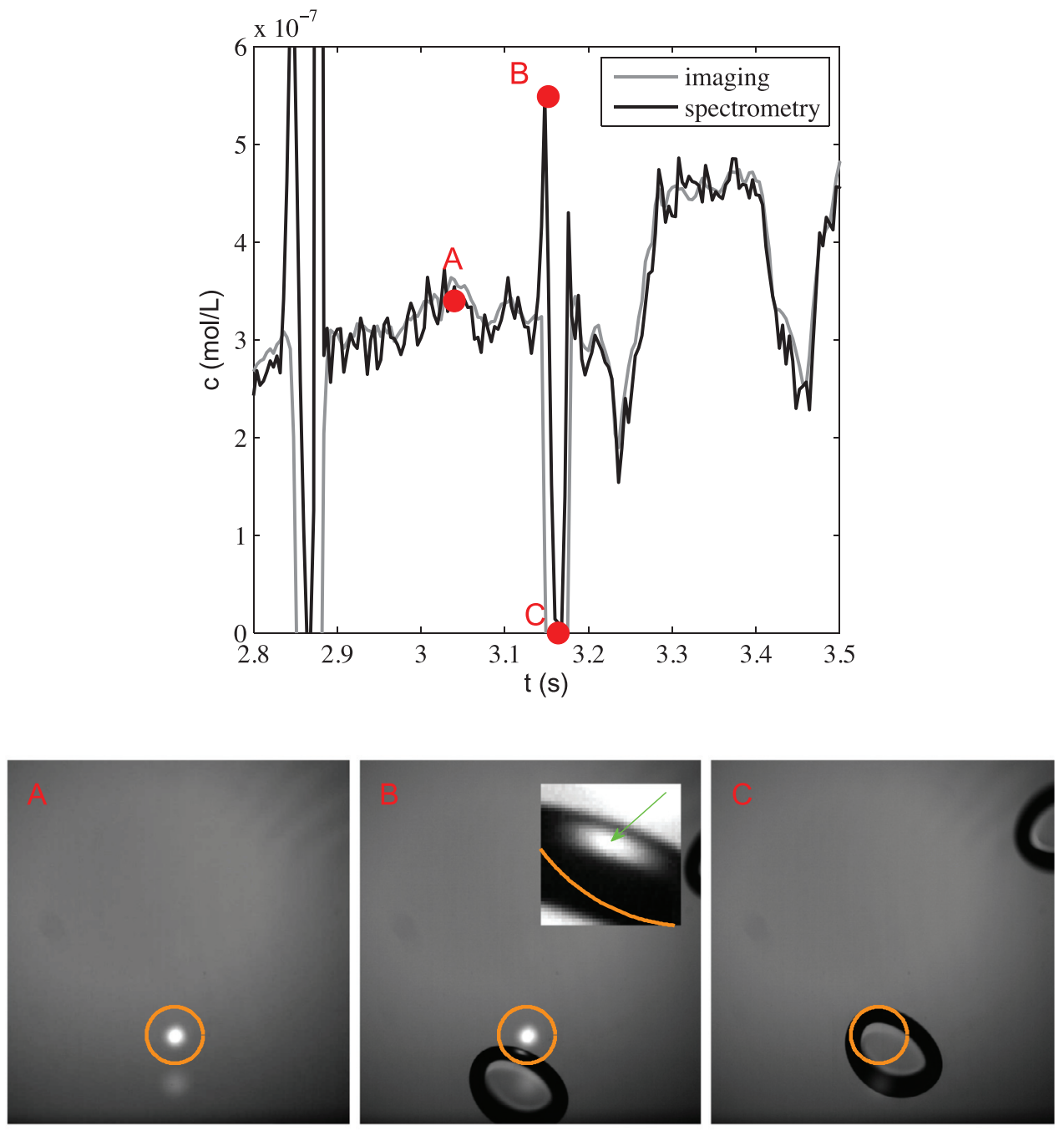

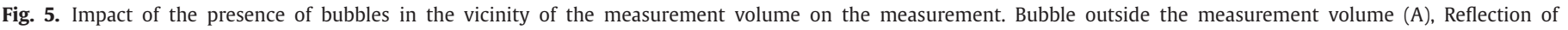

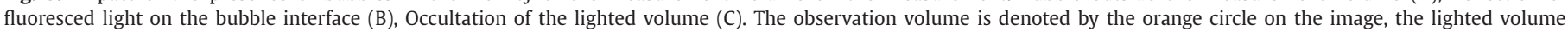
may be seen at its centre on the image in cases $\mathrm{A}$ or $\mathrm{B}$.

where $c$ is the concentration of the dye in the observation volume at time $t, K$ is a coefficient determined by calibration (see next section) and $E$ is the spectrum of the light collected by the optical fibre.

The second system combines LIF and shadowgraphy and is based on imaging. It allows to visualize the bubbles around the observation volume and simultaneously provide information about the concentration of the fluorescent dye. A red LED panel is disposed on the same side as the laser (Fig. 1). It illuminates the field around the observation volume and reveals the bubbles as shadows on the images. The spectrum of the light emitted by the LED panel shows a peak at $633 \mathrm{~nm}$ and involves only wavelengths larger than $610 \mathrm{~nm}$ which do not excite rhodamine WT (Fig. 3). It is also well separated from the maximum of intensity of the emission spectrum localised at $587 \mathrm{~nm}$. Integrating the emission spectrum only in the range of wavelength $570-610 \mathrm{~nm}$ (Eq. 1) ensures that the LED panel doesn't disturb the concentration measurement by the first system using spectrometry. On the opposite side of the cell, a camera (Photron APX RS3000) with a 105-mm optic images a field of view of $2 \times 2 \mathrm{~cm}^{2}$ around the observation volume (Fig. 1). A high-pass filter with a cut-off wavelength of $540 \mathrm{~nm}$ is placed in front of the camera to protect it from the laser beam. The camera thus registers light emitted by LIF and light from the LED panel, eventually occulted by bubble passages. The measure- ment by imaging gives two different pieces of information. First, the concentration inside the observation volume is measured by summing the grey levels of each pixels resulting from the LIF light intensity and from the constant light intensity of the LED panel. Second, the bubble distribution around the observation volume is visualized by shadow imaging in a region of $2 \times 2 \mathrm{~cm}^{2}$ illuminated by a constant light intensity of the LED panel.

\section{Validation of the concentration measurements provided by spectrometry}

Fig. 4 presents typical raw signals measured simultaneously by spectrometry (black) and by imaging (grey) for $\alpha=3.7 \%$ at $H=5$ $\mathrm{cm}$. Note that the grey levels measured by the camera have been multiplied by a constant in order to match a concentration. The comparison shows that, excepted around the very sharp peaks registered either by the spectrometer or by the camera, the raw signals measured by both different measurement techniques are remarkably similar. They show the same global time evolution of the concentration with a first global increase of the concentration from 2.5 to $4 \mathrm{~s}$ when the dye arrives at the measurement position, followed by a regular decrease towards the final concentration corresponding to a total mixing of the dye in the cell. Moreover, both signals reproduce the same rapid and intense fluctuations (around 


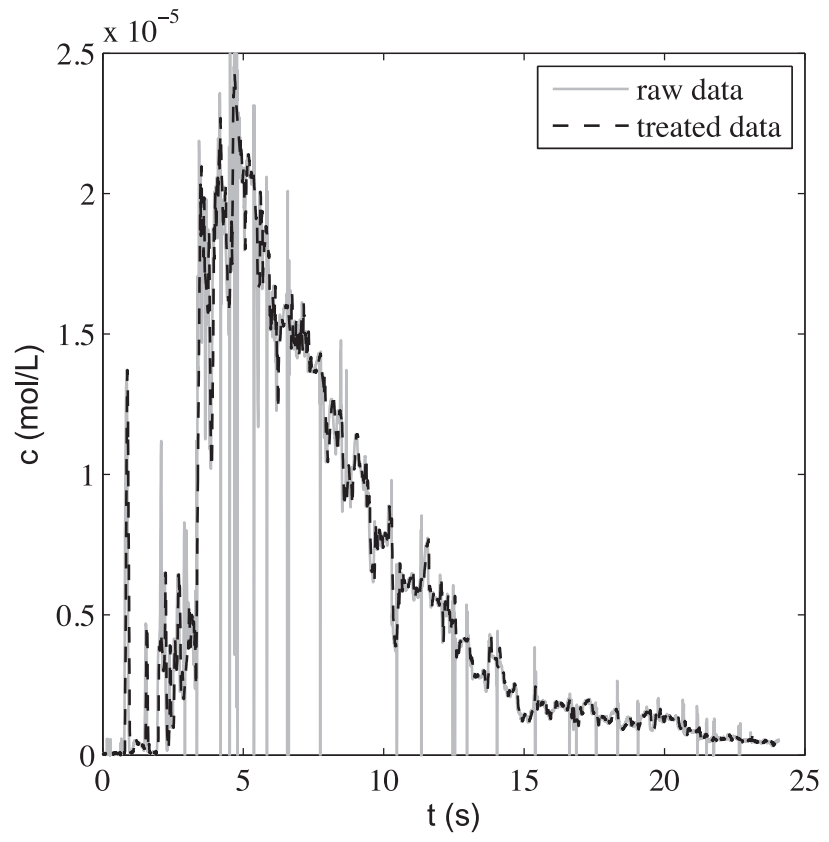

Fig. 6. Concentration measurements by spectrometry: raw data (gray), after signal processing (black). $\alpha=2.4 \% . H=10 \mathrm{~cm}$.

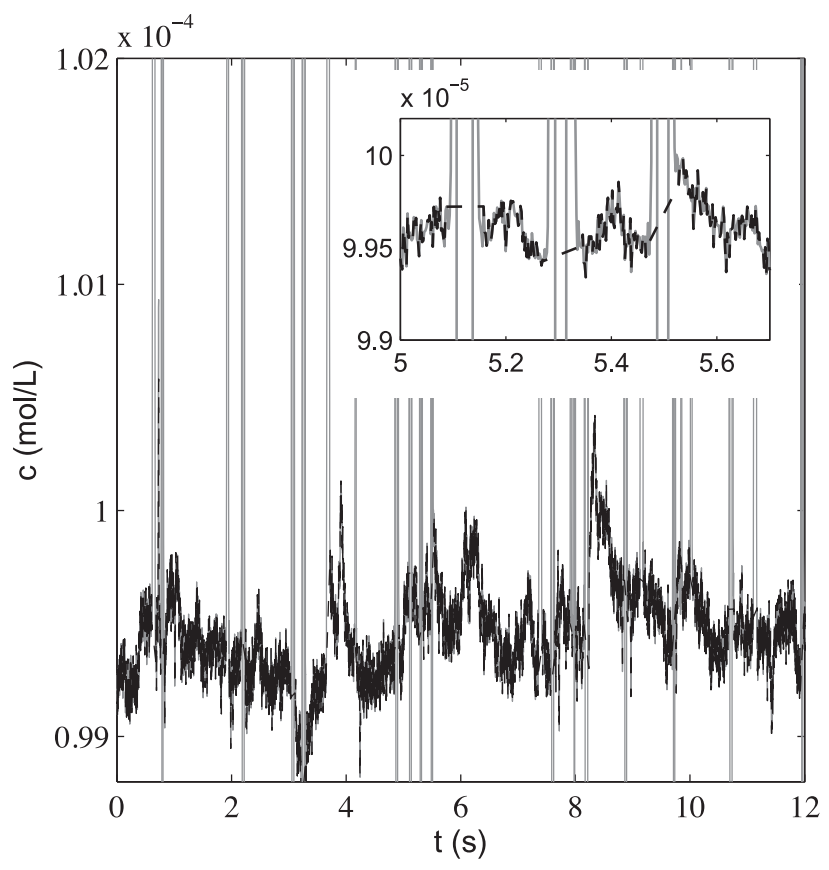

Fig. 7. Concentration measurement by LIF and spectrometry with an homogeneous concentration of rhodamine WT at $c=10^{-4} \mathrm{~mol} / \mathrm{L}$ : raw data (gray), after signal processing (black). $\alpha=5.4 \%$.

$t=4 \mathrm{~s}$ or $t=5 \mathrm{~s}$ for example) but are interrupted by strong upward or downward sudden peaks which have a characteristic time of approximately $0.05 \mathrm{~s}$. This time is very close to the duration of a bubble passage $\left(T_{b}=\frac{d}{V} \approx 0.04 \mathrm{~s}\right)$, which suggests that these strong peaks are due to the passages of the bubbles in the observation volume. This is confirmed by the simultaneous examination of both concentration signals and the distribution of the bubbles around the observation volume, which is denoted by the orange circle in Fig. 5. The distribution of the bubbles is given at three different instants denoted A to C. The case A corresponds to an instant between two strong maxima and minima, the case B (resp.

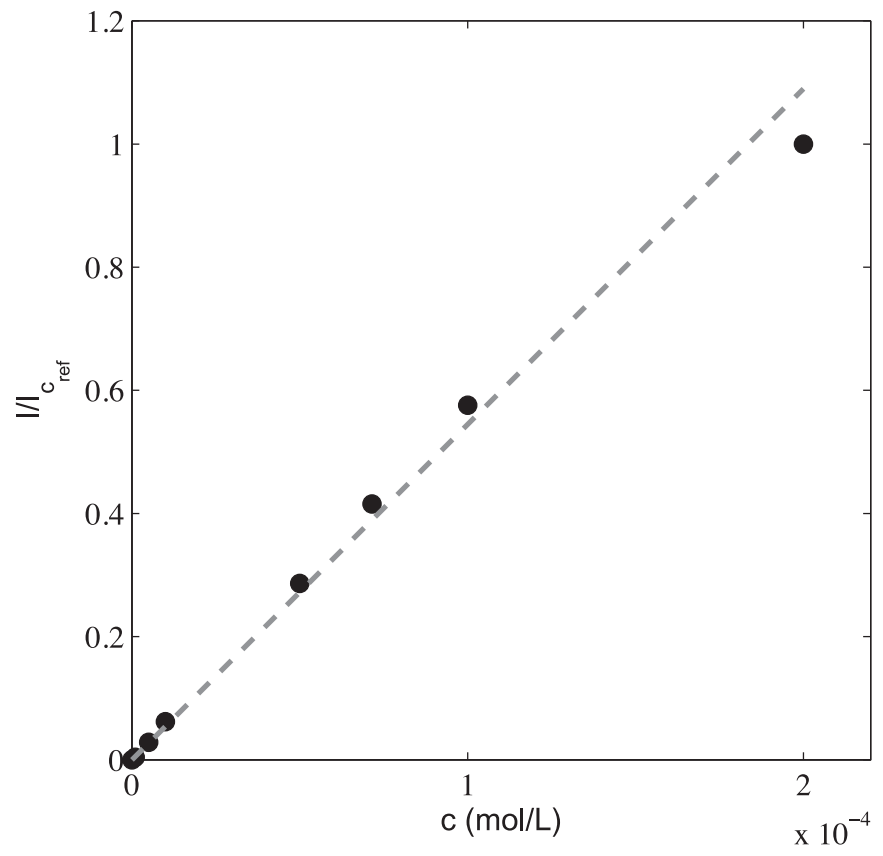

Fig. 8. Calibration of LIF measurements towards dye concentration. ( $I_{\text {cref }}$ is the intensity of the fluoresced light obtained at the concentration $c_{r e f}=2 \times 10^{-4} \mathrm{~mol} / \mathrm{L}$ ).

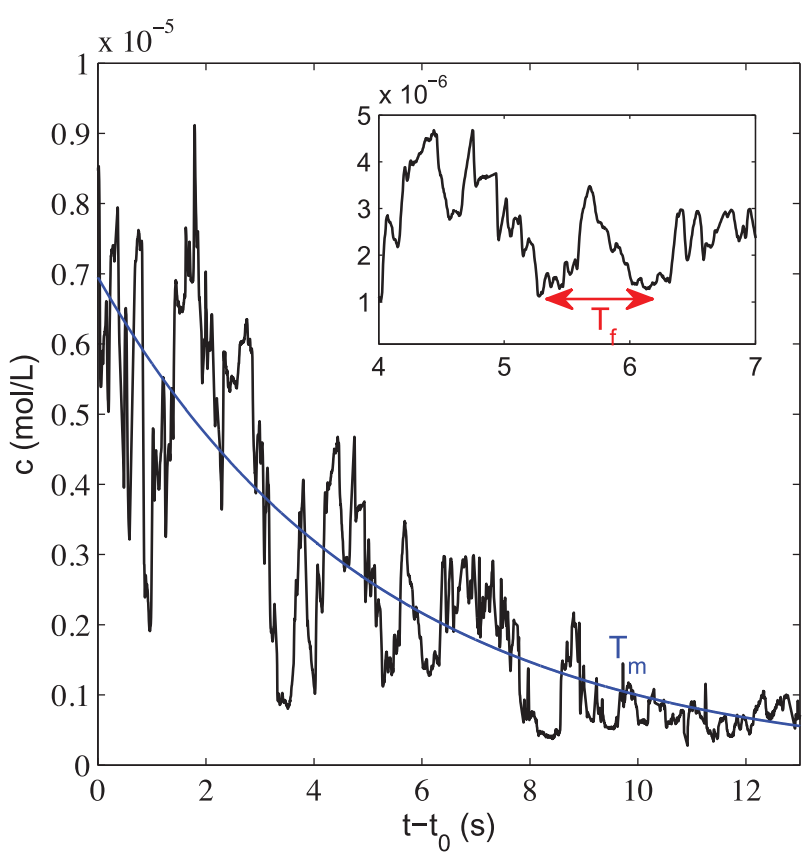

Fig. 9. Illustration of the two timescales present in the concentration signal. Signal of concentration (black). Fitting of the mean concentration by an exponential relation (Eq. 3) (blue). $\alpha=5.4 \% . H=5 \mathrm{~cm}$.

C) matches a strong maximum (resp. minimum) on the raw signal measured by the spectrometer. In case A, no bubble is present in the observation volume and the signal measured by LIF and spectrometry is therefore not disturbed by any bubbles. The measurement obtained from the spectrometer using an accurate calibration, discussed in the following, is therefore a reliable measurement of concentration. On the contrary, when bubbles are crossing the observation volume (cases B and C), strong peaks are visible. In particular, a strong upward peak on the spectrometer signal is produced by reflections and refractions of the fluoresced light at 


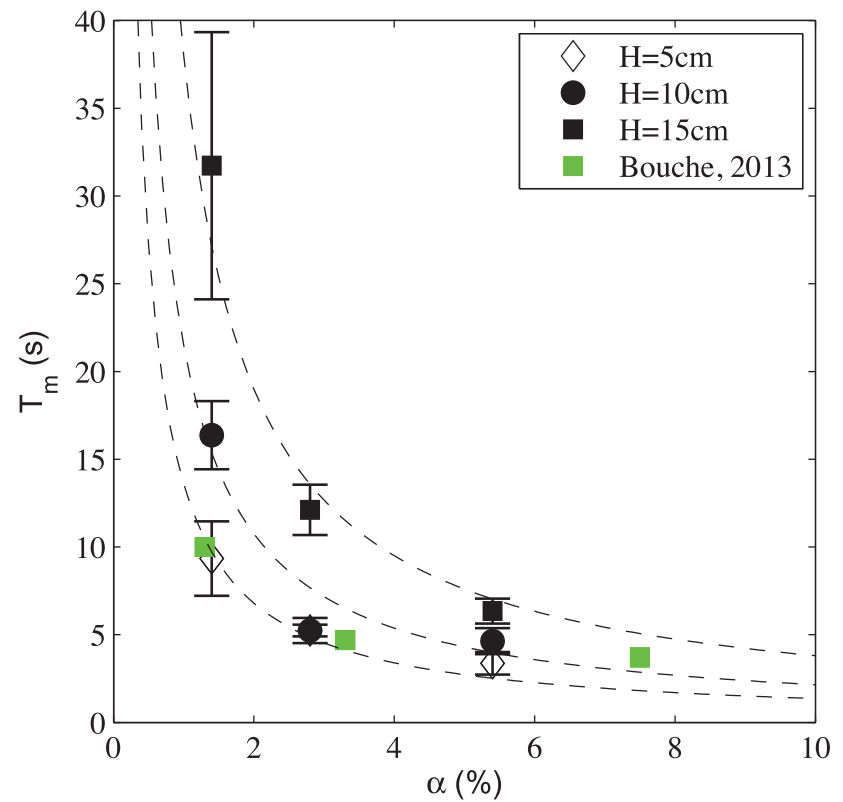

Fig. 10. Exponential decrease time as a function of the gas volume fraction for various elevations of the measurement volume. Dash lines are fits by $k / \alpha$, with $k=13.7$ $\mathrm{s}$ for $H=5 \mathrm{~cm}, k=21.5 \mathrm{~s}$ for $H=10 \mathrm{~cm}$ and $k=41.9 \mathrm{~s}$ for $H=15 \mathrm{~cm}$. (Error bars correspond to the standard deviation of individual measurements).

the bubble interface (case B). These strong reflections and refractions effects are localized on few pixels on the bubble surface (see green arrow in the insert of Fig. 5 B). The concentration measurement by imaging is thus not able to see them unlike the spectrometer. A strong downward peak is caused on both signals by the occultation of the lighted volume by a bubble (case $\mathrm{C}$ ). We can therefore conclude that these strong peaks are not representative of real fluctuations of the dye concentration and that an appropriate signal processing has to be developed to remove them.

The signal processing aims at localizing the temporal position of the spurious peaks. This operation is performed by calculating the temporal derivative of the raw signal. As the temporal derivative generated by the strong peaks are much larger than those from real concentration fluctuations, they are detected by applying a threshold to the signal derivative. A threshold equal to $\pm 2 \times 10^{-4} \mathrm{~mol} \mathrm{~L}^{-1} \mathrm{~s}^{-1}$ was suitable for all considered values of $\alpha$ and $H$. A part of the signal extending $\pm 0.012 \mathrm{~s}$ around each detected extremum has also been removed in order to localise the entire part of the signal corresponding to spurious peaks. Both the threshold and the time extension of \pm 0.012 s have been determined from the comparison of the spectrometer raw signal to the images obtained by shadow imaging. By inspecting the images, it is possible to detect precisely the start and the end of a bubble passing through the measurement volume. The corresponding part of the signal is removed and replaced by a linear interpolation. The linear interpolation is of course questionnable since the concentration is not defined in the region occupied by the bubbles. Similar methods have been used for processing liquid velocity measurements by hot-film anemometry in bubbly flows. Discussion of their consequences on the signal statistics can be found in Serizawa et al. (1983) and Suzanne et al. (1998). Here we have checked that the present interpolation has not significant influence upon PDFs of concentration fluctuations. Concerning spectra, different other techniques have been used for their determination for velocity fluctuations from signals that are interrupted by the passages of the bubbles, such as smoothing the discontinuities by a Gauss function (Lance and Bataille, 1991) or considering only intervals between bubbles where the signal is continu-
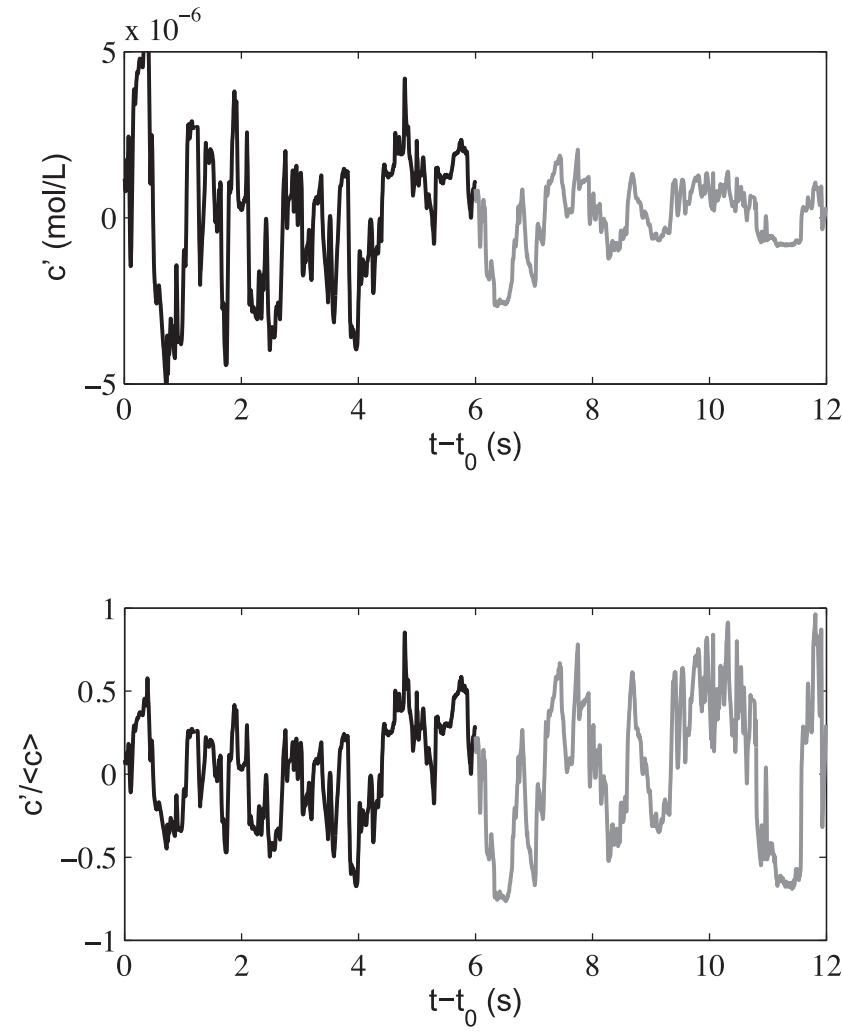

Fig. 11. Temporal evolution of the concentration fluctuations (up). After normalization by the mean concentration (down). $\alpha=5.4 \% . H=5 \mathrm{~cm}$.

ous (Martínez-Mercado et al., 2010; Bouche et al., 2014). However, all techniques eventually lead to a similar spectrum in power -3 of the wavenumber. We are therefore confident that the present signal processing preserves the characteristic of the spectrum of the concentration fluctuations. Fig. 6 presents a typical signal measured by the spectrometer before (grey curve) and after signal processing (black curve). We can see that most of the strong peaks have been removed. The perturbations induced by residual reflections or partial occultations have been quantified by applying the signal processing to a situation where the Hele-Shaw cell is filled with a homogeneous concentration of rhodamine WT at $c_{0}=10^{-4}$ $\mathrm{mol} / \mathrm{L}$ and $\alpha=5.4 \%$ (Fig. 8). The processed signal (black curve) is obviously more regular than the raw signal (grey curve) but still shows some fluctuations due to residual perturbations. A minor amount of them comes from the fact that the observation volume is much larger than the lighted volume. The signal-to-noise ratio could therefore be slightly enhanced by having a smaller observation volume, which must remain in any case larger than the lighted volume. The residual perturbations can be quantified by the standard deviation of the signal after processing (Fig. 8). For this most critical case, the standard deviation is equal to $2.1 \times 10^{-7} \mathrm{~mol} / \mathrm{L}$, which corresponds to $2 \times 10^{-3} c_{0}$. Residual perturbations are therefore negligible and reliable concentration measurements are performed in a confined bubbly flow for gas volume fractions up to $5.4 \%$.

A calibration of the measurement by LIF and spectrometry has been carried out to determine the range of validity of Eq. 1 in terms of concentration and to estimate the proportionality coefficient $K$. It has been achieved by filling the Hele-Shaw cell with an homogeneous solution of rhodamine WT at a given concentration and by calculating the mean intensity of the fluoresced light measured by LIF after signal processing. Fig. 7 shows the calibration curve for dye concentrations ranging from $c=0$ to $c=2 \times 10^{-4}$ 

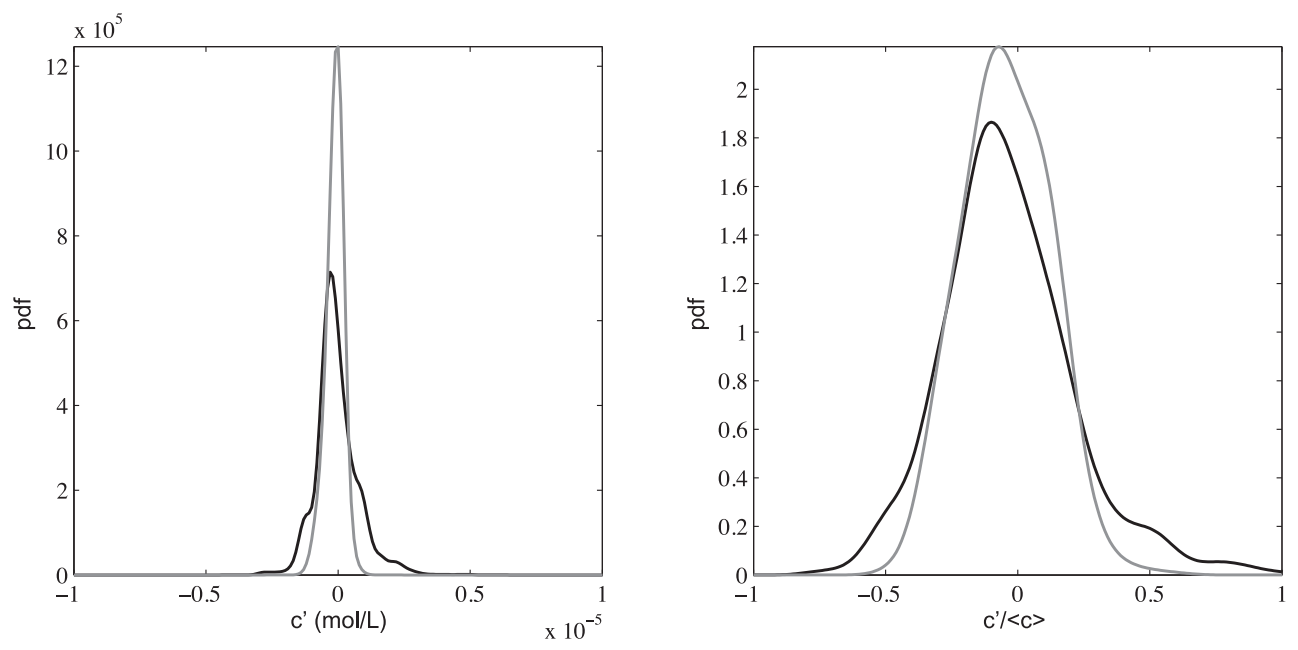

(a) $\alpha=1.2 \%$
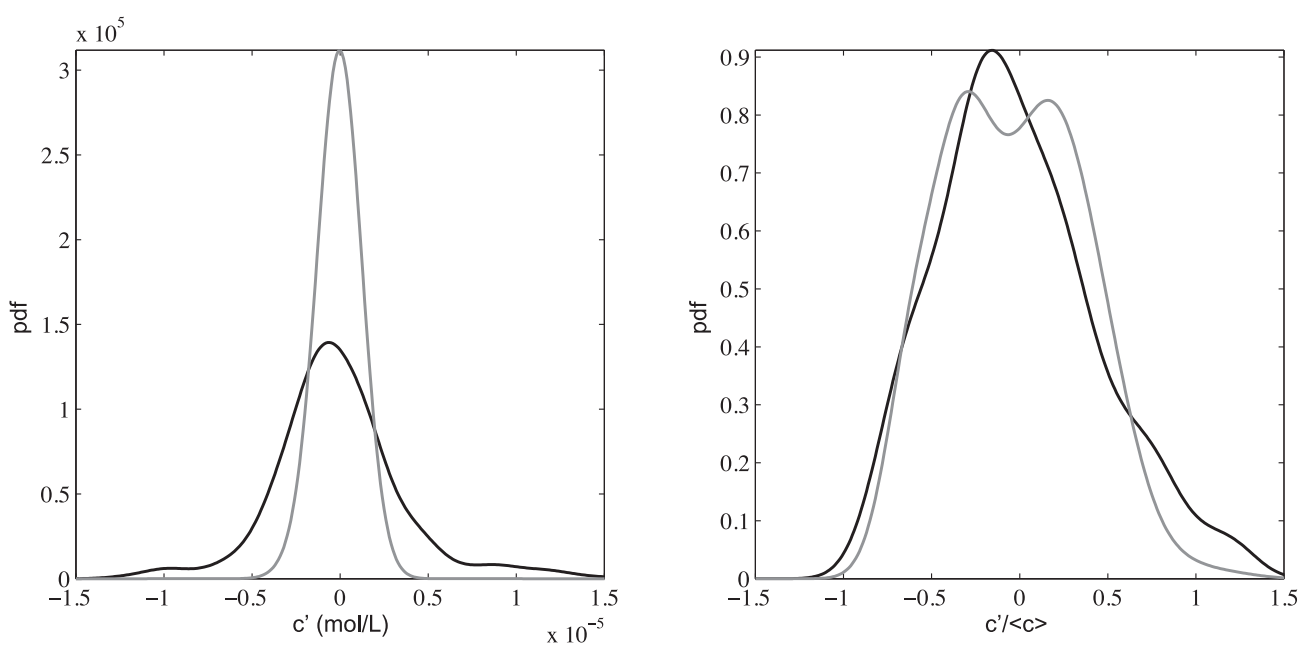

(b) $\alpha=5.4 \%$

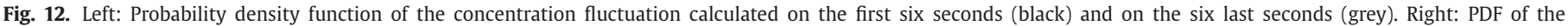
fluctuations normalised by the mean concentration. Results obtained from 4 independent runs at $H=5 \mathrm{~cm}$.

$\mathrm{mol} / \mathrm{L}$. Note that the intensity of the fluoresced light $I$ has been normalized by the intensity of the fluoresced light measured at concentration $c_{\text {ref }}=2 \times 10^{-4} \mathrm{~mol} / \mathrm{L}$. The intensity of the fluoresced light evolves linearly for concentrations lower than $c=1 \times 10^{-4}$ mol/L. Eq. 1 is thus valid for the whole range of concentration considered here.

In general, the intensity of the fluoresced light depends also on laser intensity, $\mathrm{pH}$ and temperature. Concerning the laser intensity, it has been checked by using neutral density filter that the intensity of the fluoresced light evolves linearly with the laser intensity. Moreover, the Fig. 8 shows that the potential variations of the measured concentration induced by fluctuations of the laser intensity can be neglected and that no photobleaching occurs. Possible $\mathrm{pH}$ variations can also been disregarded in the present case since all the experiments have been conducted with the same aqueous solution. Finally, the temperature has been recorded during each mixing experiments and only varies within 20.0 to $20.8{ }^{\circ} \mathrm{C}$. Temperature variations were therefore too small to significantly influence the measurements.

\section{Results}

The maximum of concentration is reached at an instant that depends on the gas volume fraction $\alpha$ and the elevation $H$ of the measuring point. This maximum is detected for each considered case and only the decreasing part of the concentration signal, which is not influenced by the details of the initial injection conditions, is considered hereafter. Fig. 9 shows a typical time evolution of the concentration measured by the spectrometer for $\alpha=5.4 \%$ and $H=5 \mathrm{~cm}$. Two main features, involving two different times scales, are clearly visible. The first one is a global decrease of the concentration on a time $T_{m}$ of the order of ten seconds. This slow decay has been checked to be reproducible for different tests performed at the same $\alpha$ and $H$. The second one is the presence of intense and random fluctuations characterized by a timescale $T_{f}$ of the order of a few tenths of a second. The concentration signal $c$ is thus the sum of a slowly time-varying mean concentration $\langle c\rangle$ and random fluctuations $c^{\prime}$ :

$c(t)=\langle c\rangle(t)+c^{\prime}(t)$. 
For each independent run, the time evolution of the mean concentration is well described by a decreasing exponential function (Fig. 9),

$\langle c\rangle(t)=c\left(t_{0}\right) \exp \left(-\frac{t-t_{0}}{T_{m i}}\right)$

where $t_{0}$ is the instant where the maximum of concentration is reached and $T_{m i}$ is the characteristic time of decrease for a single run $i$. For each $\alpha$ and $H$, the mean characteristic time of decrease $T_{m}$ is calculated by averaging the times $T_{m i}$ over the five independent runs and error bars are estimated from the standard deviation of individual measurements. Fig. 10 shows that $T_{m}$ depends on both $\alpha$ and $H$. At a given $H, T_{m}$ decreases with the gas volume fraction, which is not surprising since the mixing efficiency is expected to be an increasing function of $\alpha$. It is well described by the function $k / \alpha$ (dash lines in Fig. 10), with $k=13.7 \mathrm{~s}$ for $H=5 \mathrm{~cm}$, $k=21.5 \mathrm{~s}$ for $H=10 \mathrm{~cm}$ and $k=41.9 \mathrm{~s}$ for $H=15 \mathrm{~cm}$. For a given $\alpha, T_{m}$ increases with $H$ and is multiplied by a factor 2 between $H=5 \mathrm{~cm}$ and $H=15 \mathrm{~cm}$, indicating that the mixing process slows down when the distance from the injection increases. The dependence of $T_{m}$ with the distance $H$ has to be linked to the diminution of the concentration gradients. The timescale of mixing depends both on the structure of the bubbly flow and on the gradient of concentration. For a normal diffusive process, the timescale would be given by $\Delta^{2} / D$. The diffusion coefficient $D$ depends on the flow structure and is therefore independent of the elevation $H$. However, the square length, $\Delta^{2}=\left(\frac{1}{C} \frac{d^{2} C}{d x^{2}}\right)^{-1}$, increases with $H$ as concentration gradients decrease. Even if the mixing in a Hele-Shaw cell cannot be described by a normal diffusion process, it is expected that the timescale $T_{m}$ increases as the gradient of concentration diminishes. In the same cell but by means of a planar laser induced fluorescent method with two dyes, Bouche et al. (2013) have measured the time evolution of the total mass of dye contained within a window of $115 \times 70 \mathrm{~mm}^{2}$ located at $H=4 \mathrm{~cm}$. They have also found an exponential decrease of this global concentration. Their result obtained in a large measurement window (also shown in Fig. 10) is very close to the present local measurement obtained at $H=5 \mathrm{~cm}$, indicating that the size of the measurement window has a weak influence on the mean decrease of the concentration. Bouche et al. (2013) have shown that the observed exponential decay is incompatible with a purely diffusive process and have concluded that the capture and the advection of the dye within the bubble wakes is probably the main mechanism responsible for the mixing in a confined cell. This remains to be confirmed by timeresolved measurements of the local concentration.

The following analysis of the fluctuations of concentration is performed by considering measurements recorded at $H=5 \mathrm{~cm}$ where the dynamics of the signals remains good over a long enough period of time. Statistics are carried out over 4 independent runs of $12 \mathrm{~s}$, which allows to get a reasonable statistical convergence of PDFs and spectra. Instantaneous fluctuations of the concentration are obtained by subtracting the mean concentration of each independent test determined by the exponential fitting (Eq. 3) to the instantaneous signal of concentration for $t \geq t_{0}$ :

$c^{\prime}(t)=c(t)-c\left(t_{0}\right) \exp \left(-\frac{t-t_{0}}{T_{m i}}\right)$.

The upper Fig. 11 shows the temporal evolution of the concentration fluctuations for a typical case at $\alpha=5.4 \%$. Similarly to the mean concentration, the amplitude of the fluctuations decreases. However, we see in the lower Fig. 11 that, once normalized by the mean concentration, the amplitude of the fluctuations seems no longer evolving with time. This can be checked by comparing the probability density functions (PDFs) of the fluctuations determined from the first half of the signal $\left(0 \leq t-t_{0} \leq 6 \mathrm{~s}\right)$ to that determined from the second part $\left(6 \mathrm{~s} \leq t-t_{0} \leq 12 \mathrm{~s}\right)$. Fig. 12 shows the PDFs

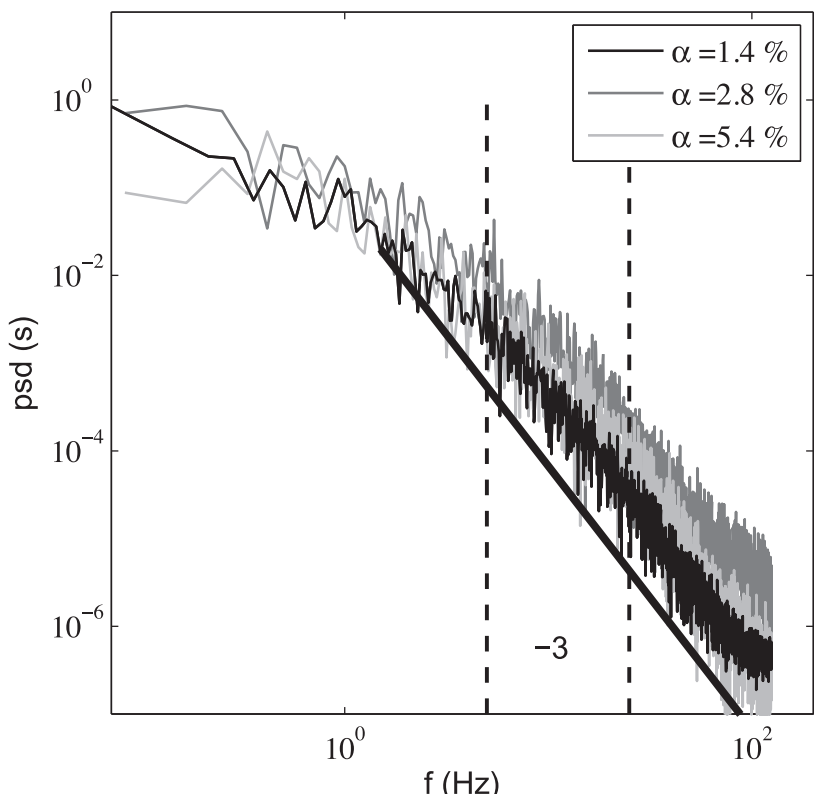

Fig. 13. Spectra of the normalized fluctuations of concentration for various $\alpha$ at $H=5 \mathrm{~cm}$. The vertical dash lines show the spectral range [5-25 $\mathrm{Hz}]$ where a -3 slope is observed.

averaged over 4 independent tests of both the raw (on the left) and the normalized (on the right) concentration fluctuations for two different gas volume fractions ( $\alpha=1.2 \%$ and $\alpha=5.4 \%$ ). While the width of the raw PDFs are very different, it turns out that the normalized PDFs of the two parts of the signal nicely match. Provided they are rescaled by the slowly time-evolving mean concentration, we can thus conclude that the fluctuations of concentration are statistically stationary in time. Moreover, the PDF of concentration fluctuations present the same properties as the one of vertical velocity fluctuations of the liquid (Bouche et al., 2014). Both are non-Gaussian with strong positive fluctuations more probable than strong negative fluctuations. In particular, this implies that the upward transport of the dye is different from the downward transport. It is therefore not compatible with a normal diffusion process which would be characterized by a symmetric transport in the vertical direction. For liquid fluctuations, the long tail on the side of upward fluctuations is known to be the signature of the bubble wakes. Here, the tail observed for positive concentration fluctuations is thus probably related to patches of dye that are trapped and entrained in the bubble wakes.

A spectral analysis of the concentration fluctuations has also been carried out for different $\alpha$ and $H$. Fig. 13 shows the spectra of the normalized concentration fluctuations averaged over 4 independent runs of $12 \mathrm{~s}$ each for the 3 studied gas volume fractions $\alpha$ at $H=5 \mathrm{~cm}$. It is observed to be weakly dependent of $\alpha$ and $H$ and to show, in any case, an evolution as the power -3 of the frequency in between 5 and $25 \mathrm{~Hz}$. The spatial spectrum of the liquid velocity fluctuations, measured by Bouche et al. (2014), showed a similar subrange in power -3 of the wavenumber for wavelengths in between $d$ and $5 d$, which correspond to the same frequencies as observed here: $\frac{V}{d} \approx 5 \mathrm{~Hz}$ and $\frac{V}{5 d} \approx 25 \mathrm{~Hz}$. The timescales of concentration fluctuations are therefore closely related to those of the liquid velocity fluctuations. Concerning the liquid fluctuations, Risso (2011) has shown that such a spectral behaviour can be interpreted by a superposition of random disturbances induced by each individual bubble. Thus, if we assume that the mixing is controlled by the capture, the transport and the release of the dye by the wake of each bubble, the same interpretation is expected to be also valid for the concentration fluctuations. 
Finally, we can conclude from the analysis of the fluctuations that the mixing in a confined bubbly flow is controlled by the agitation in the liquid phase and is due to random passages of finitesize patches of dye.

\section{Conclusion}

A new technique based on laser induced fluorescence and spectrometry has been developed to measure the local instantaneous concentration of a solute within a bubbly flow. It involves a specific signal processing to get rid of spurious peaks caused by the presence of bubbles in the vicinity of the measurement volume. Thanks to comparisons with a technique based on high-speed imaging, the method has been validated and proved to provide reliable measurements with a time resolution of $4 \mathrm{~ms}$. Time-resolved measurements of the dye concentration have thus been obtained, giving access for the first time to an accurate description of local fluctuations of concentration in a bubbly flow.

The technique has been applied to the investigation of the mixing of a passive dye initially injected at a given position within a homogeneous bubbly flow confined in a thin gap cell. Time records of the concentration have been obtained at various distances above the dye injection point and for various gas volume fractions. The concentration signal involves a mean component that slowly evolves in time and rapid random fluctuations that are the signature of the passages of patches of dye in the measurement volume.

As already observed for the global evolution of the total mass of dye contained within a large measurement volume by Bouche et al. (2013), the mean concentration decays in time by following an exponential law. The timescale of decay is observed to increase with the distance from the injection point and to decrease with the gas volume fraction. It also seems to be weakly dependent on the size of the measurement volume.

The fluctuations of concentration have been analysed in both time and frequency domains. Once normalized by the mean concentration, they turn out to be statistically stationary and involve timescales similar to those of the velocity fluctuations generated in the vicinity of the bubbles. Their PDF has an asymmetric shape similar to that of the vertical liquid fluctuations, with a tail for large positive fluctuations. This suggests that the transport of dye is different in the upward and downward direction and is not compatible with a purely diffusive process of mixing. The spectrum of the fluctuations shows an evolution in power -3 of the frequency in the same range as the spectrum of the velocity fluctuations previously measured by Bouche et al. (2014). As for the liquid velocity fluctuations (Risso 2011), the concentration can thus be interpreted as a collection of random patches of dyes of various sizes. This reveals a peculiar mechanism of mixing which is strongly intermittent and convective.

Future work should be devoted to derivation of a physical mixing model based on the capture, transport and release of the dye in the bubble wakes. The application of the present technique to investigate the fluctuations of concentration in a three-dimensional dense bubble column is also planed.

\section{Acknowledgements}

The authors would like to dedicate this work to the memory of the late Emmanuella Bouche.

\section{References}

Alméras, E., Risso, F., Roig, V., Cazin, S., Plais, C., Augier, F., 2015. Mixing by bubbleinduced turbulence. J. Fluid Mech. 776, 458-474. doi:10.1017/jfm.2015.338.

Amoura, Z., 2008. Etude hydrodynamique de l'écoulement traversant un réseau alléatoire de sphères fixes. Institut Nationale Polytechnique de Toulouse Ph.D. thesis.

Besnaci, C., 2012. Mélange induit par un écoulement au travers un réseau aléatoire d'obstacles. Institut Nationale Polytechnique de Toulouse Ph.D. thesis.

Besnaci, C., Roig, V., Risso, F., 2010. Mixing induced by a random dispersion at high particulate Reynolds number. In: Proceedings of 7 th International Conference on Multiphase Flow, Tampa.

Bouche, E., Cazin, S., Roig, V., Risso, F., 2013. Mixing in a swarm of bubbles rising in a confined cell measured by mean of PLIF with two different dyes. Exp. Fluids 54, 1552. doi:10.1007/s00348-013-1552-0.

Bouche, E., Roig, V., Risso, F., Billet, A.-M., 2012. Homogeneous swarm of highReynolds-number bubbles rising within a thin gap. part 1. bubble dynamics. J. Fluid Mech. 704, 211-231. doi:10.1017/jfm.2012.233.

Bouche, E., Roig, V., Risso, F., Billet, A.-M., 2014. Homogeneous swarm of highReynolds-number bubbles rising within a thin gap. part 2. liquid dynamics. J. Fluid Mech. 758, 508-521. doi:10.1017/jfm.2014.544.

Filella, A., Ern, P., Roig, V., 2015. Oscillatory motion and wake of a bubble rising in a thin-gap cell. J. Fluid Mech. 778, 60-88. doi:10.1017/jfm.2015.355.

Lance, M., Bataille, J., 1991. Turbulence in the liquid phase of a uniform bubbly air? Water flow. J. Fluid Mech. 222, 95-118. doi:10.1017/S0022112091001015.

Martínez-Mercado, J., Chehata Gómez, D., Van Gils, D., Sun, C., Lohse, D., 2010. On bubble clustering and energy spectra in pseudo-turbulence. J. Fluid Mech. 650, 287-306. doi:10.1017/S0022112009993570.

McClure, D.D., Aboudha, N., Kavanagh, J.M., Fletcher, D.F., Barton, G.W., 2015. Mixing in bubble column reactors: Experimental study and CFD modeling. Chem. Eng. J. 264, 291-301. doi:10.1016/j.cej.2014.11.090.

Pandit, A., Joshi, J., 1983. Mixing in mechanically agitated gas-liquid contactors, bubble columns and modified bubble columns. Chem. Eng. J. 38, 1189-1215

Riboux, G., Legendre, D., Risso, F., 2013. A model of bubble-induced turbulence based on large-scale wake interactions. J. Fluid Mech. 719, 362-387. doi:10.1017/ jfm.2013.12.

Risso, F., 2011. Theoretical model for k-3 spectra in dispersed multiphase flow. Phys. fluids 23, 011701.

Risso, F., Roig, V., Amoura, Z., Billet, A.-M., 2010. The dual nature of pseudo-turbulence analyzed from spatial and time avergings of a flow through random obstacles. Proceedings of the 7th International Conference on Multiphase Flow, Tampa.

Roig, V., Roudet, M., Risso, F., Billet, A.-M., 2012. Dynamics of a high-Reynoldsnumber bubble rising within a thin gap. J. Fluid Mech. 707, 444-466. doi:10. 1017/jfm.2012.289.

Serizawa, A., Tsuda, K., Michiyoshi, I., 1983. Real-time measurement of two-phase flow turbulence using dual-sensor anemometry. IUTAM Symposium of Measuring Techniques in Gas-Liquid Two-phase Flows, Nancy, France.

Suzanne, C., Ellingsen, K., Risso, F., Roig, V., 1998. Local measurements in turbulent bubbly flows. Nucl. Eng. Des. 184, 319-327.

Tanino, Y., Nepf, H.M., 2008. Lateral dispersion in random cylinder arrays at high Reynolds number. J. Fluid Mech. 600, 339-371. doi:10.1017/S0022112008000505.

White, B.L., Nepf, H.M., 2003. Scalar transport in random cylinder arrays at moderate Reynolds number. J. Fluid Mech. 487, 43-79. doi:10.1017/ S0022112003004579. 